\title{
Technikfolgenabschätzung für die Gesellschaft der singularitäten
}

\author{
Neue Impulse aus kulturtheoretischer sicht am Beispiel \\ von Offenen Werkstätten
}

\author{
Lorenz Erdmann, Competence Center Foresight, Fraunhofer-Institut für System- und Innovationsforschung ISI, Breslauer Str. 48, \\ 76139 Karlsruhe (lorenz.erdmann@isi.fraunhofer.de), (1) orcid.org/0000-0002-4893-780X \\ Bastian Lange, Lehrstuhl für Wirtschaftsgeographie und Regionalentwicklung, Universität Vechta (bastian.lange@uni-vechta.de), \\ (1) orcid.org/0000-0003-3619-900X
}

Der Kulturwissenschaftler Andreas Reckwitz hat Prozesse der Singularisierung der Gesellschaft diagnostiziert, in denen sich die Eigenschaft des Besonderen massenhaft nicht nur auf menschliche Subjekte, sondern auch auf Orte, Ereignisse, Kollektive und Objekte erstreckt. Die epistemischen Implikationen der sich so verändernden Gesellschaft für die Technikfolgenabschätzung (TA) wurden bislang wenig beachtet. Wir argumentieren anhand des Beispiels der Singularitätseigenschaften von Offenen Werkstätten, dass eine konzeptionelle Befassung der TA mit den gesellschaftlichen Effekten von Singularitäten notwendig ist. Wir stellen daher neue Eckpunkte für die TA-Programmatik zur Diskussion, die auch Singularisierungstechniken wie z. B. den 3-D-Druck betrifft: (1) die Wahrnehmung des Besonderen als Relevantes, (2) die Abbildung der Kulturalisierung von Wirtschaft und Gesellschaft und (3) realitätsangemessenes Ausschneiden von Systemen aus dem kulturalisierten Supersystem.

\section{Technology assessment for the society of singularities}

New impulses from a cultural theory perspective using the example of open workshops

Cultural scientist Andreas Reckwitz has recently diagnosed processes of singularization of society, in which the quality of being special not only applies en masse to human subjects, but also to places, events, collectives, and objects. The epistemic implications of these societal changes for technology assessment (TA) have hardly been addressed to date. Building upon the singular features of open workshops, we argue that TA needs to develop new concepts for assessing the societal impact of singularities, and suggest three key points that also apply to other sin-

This is an article distributed under the terms of the Creative Commons Attribution License CCBY 4.0 (https://creativecommons.org/licenses/by/4.0/)

https://doi.org/10.14512/tatup.28.1.65

Submitted: 13.07.2018. Peer reviewed. Accepted: 30. 01.2019 gularization technologies, such as 3D printing: (1) perception of the special nature as being relevant, (2) representation of the culturalization of economy and society, and (3) adequate separation of systems from the culturalized super system.

Keywords: singularization, open workshops, cultural theory, TA concepts

\section{Offene Werkstätten als Singularitäten - ein Gegenstand für die TA}

In neueren theoretischen Reflexionen wird die zeitdiagnostische Bindung der TA zwar anhand vergangener Zeitkontexte thematisiert (Alpsancar 2018), aktuelle Zeitdiagnosen und ihre Einschreibung in die Technikfolgenabschätzung (TA) fehlen jedoch in der Debatte. Die TA modelliert verschiedene Gegenstandbereiche wie Technologie, Organisationsweise, Gebrauch und ihre Folgen mit unterschiedlichen Systembeschreibungen (Kornwachs 2018), wobei das abgegrenzte System als Teil eines Supersystems verstanden werden muss (Ropohl 2012, S. 73-74).

Mit der Singularisierung der Gesellschaft unternimmt Andreas Reckwitz (2017) eine Zeitdiagnose, die eine kulturtheoretische Verortung von abgegrenzten Systemen erlaubt. ${ }^{1}$ Die TA hat sich den technischen Phänomenen der Gesellschaft der Singularitäten bislang nur punktuell angenommen, wie zum Beispiel den Umweltwirkungen von personalisierter Fertigung (Kohtala und Hyysalo 2015). Die Einbettung von Untersuchungen in ein kulturalisiertes Supersystem, wie es mit der Theorie der Gesell-

1 Siehe auch die Rezension des Buches in dieser Zeitschrift (Frank 2018). 


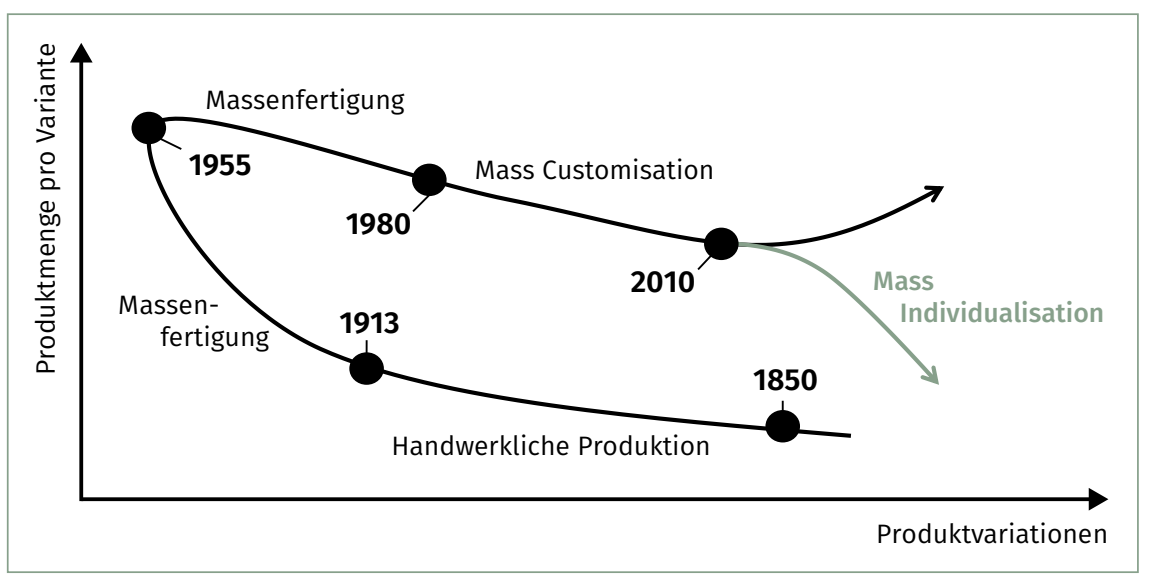

Abb.1: Produktionsparadigmen im Wandel.

Quelle: Eigene Darstellung in Anlehnung an Koren et al. (2015) und Simons et al. (2016)

grundsätzlich allen Zugang zu Arbeitsräumen, Know-how, Werkzeugen und Maschinen wie Stichsägen, Laser-Cutting und 3-D-Drucker bieten (Lange et al. 2016). An solchen dauerhaft oder temporär genutzten Orten sind Menschen selbstbestimmt, entweder in Gemeinschafts- oder in Eigenarbeit, handwerklich, maschinell oder künstlerisch tätig. Offene Werkstätten gibt es bereits für eine große Bandbreite an Gewerken, häufig auch - im Gegensatz zum konventionellen Handwerk - im Verbund: Nähwerkstätten, Fab-Labs und Reprographie (Papierdruck/3-D-Druck/Foto), Holz-, Metall- und Kunststoffverarbeitung, Möbelbau, Lastenfahrradbau, Elektronikwerkstätten, Repair-Cafés etc. Hierbei

schaft der Singularitäten formuliert wird, bedeutet somit eine wichtige Ergänzung der TA. Alternative Zeitdiagnosen wie die McDonaldisierung der Gesellschaft (Ritzer 2017) verdeutlichen die Notwendigkeit, kulturtheoretische Verortungen gut begründet, ggf. auch kontingent, vorzunehmen.

Andreas Reckwitz definiert Singularitäten als „Entitäten, die innerhalb von sozialen Praktiken als besonders wahrgenommen und bewertet, fabriziert und behandelt werden" (Reckwitz 2017, S. 50-51). Mit Entitäten sind Menschen, Orte, Ereignisse, Kollektive und Objekte gemeint. Prozesse der Singularisierung habe es seit der Renaissance immer wieder gegeben, in der Spätmoderne jedoch in nie da gewesener Quantität und Qualität. Wesentliche Triebkraft sei, so Reckwitz, die neue Mittelklasse, die Berufe in der Kultur- und Wissensökonomie ausübt und zur emotionalen Befriedigung ihres Authentizitätswunsches zunehmend Singularitätsgüter nachfragt. Diese Nachfrage wird durch Stimulierung des Begehrens seitens der kulturalisierten Ökonomie weiter dynamisiert (Böhme 2016).

In der Gesellschaft der Singularitäten verschiebt sich das Produktionsparadigma von der Massenfertigung und der mass customisation jetzt zur mass individualisation, wie Abbildung 1 zeigt. Im Zuge der Produktindividualisierung steigt die Produktvielfalt und gleichzeitig nimmt die produzierte Menge pro Produktvariante ab. Singuläre Güter werden derzeit handwerklich oder mit 3-D-Druckern produziert und über Vertriebsportale wie Etsy und eBay gehandelt. Unter dem Leitbild ,Losgröße 1 werden Produktion und Vertrieb singulärer Güter auch von kommerziellen Großakteuren anvisiert (Bogner et al. 2018). Reckwitz (2017) weist solchen und anderen Singularitäten eine strukturbildende Kraft für Wirtschaft, Arbeit, Technologie, Lebensstile und Alltagskultur zu. Ihm zufolge treten anstelle weniger großer Erzählungen in der Moderne unzählige kleine Erzählungen in der Spätmoderne.

Als einen Ausdruck der kulturtheoretischen Singularitätsthese von Reckwitz verstehen wir die Praktiken in Offenen Werkstätten. Offene Werkstätten sind Infrastrukturen, die produzieren, modifizieren und reparieren die Nutzer der Offenen Werkstätten besondere Objekte wie zum Beispiel Lastenfahrrad-Unikate, Saftpressen und Radios aus alter Elektronik. Damit steht die wachsende Zahl an Aktivitäten in Offenen Werkstätten (Lange et al. 2016) sinnbildlich auch für eine mächtigere These zur gesellschaftlichen Entwicklung: nämlich die Aufwertung des Besonderen (Pfaller 2018; Reckwitz 2017). Technologien in der Logik des Allgemeinen (Maschinen, Grundstoffe etc.) werden nach Reckwitz (2017, S. 103) immer mehr zu einer Infrastruktur für die Fabrikation von Besonderheiten.

Offene Werkstätten dienen uns im Folgenden als Beispiel zur Verdeutlichung der methodischen Schwierigkeiten und Entwicklungsbedarfe für eine TA über singuläre Phänomene. Hierzu charakterisieren wir zunächst das Phänomen der Offenen Werkstätten, diskutieren dann mögliche Implikationen ihres Singularitätscharakters für die TA und schließen mit Desiderata für eine neue TA-Agenda im Verhältnis zur wachsenden Bedeutung singulärer Phänomene, als komplementäre Sichtweise zu den weitverbreiteten generalisierenden Konzepten der TA.

\section{Bestandsaufnahme von Offenen Werk- stätten in Deutschland}

Im Forschungsprojekt COWERK ${ }^{2}$ wurden unter unserer Mitwirkung die Strukturen und Praktiken von Offenen Werkstätten in Deutschland mit Hilfe von teilnehmender Beobachtung und einer standardisierten Online-Befragung von 453 Offenen Werkstätten im Jahr 2015 erhoben. Viele Offene Werkstätten sind über Internetrecherchen nicht zu identifizieren, dem Anschein nach sind sie jedoch vielerorts im Stadtteil, im Dorf, in

2 Das Projekt Commons-based Peer Production in Offenen Werkstätten (COWERK) wurde vom Bundesministerium für Bildung und Forschung im Schwerpunktprogramm „Nachhaltiges Wirtschaften“ gefördert (Förderkennzeichen 01UT1401). Forschungsverbund: IÖW, Fraunhofer UMSICHT, Fraunhofer ISI, Universität Bremen, Multiplicities, Verbund Offener Werkstätten. 
der Nachbarschaft integriert - oder sie wirken im Stillen oft auch unter anderem Namen. Derzeit dürfte es in Deutschland geschätzte 1.000 Offene Werkstätten geben, Tendenz steigend.

Die Auswertung der 103 erhaltenen Antworten der Online-Befragung zeigt (Lange et al. 2016; Lange 2017), dass es sich bei $41 \%$ der Werkstätten um eingetragene Vereine handelt, wohingegen $33 \%$ explizit informell zusammengeschlossen sind. Ihre Werkstattgröße reicht von 10 bis 1.500 Quadratmetern, der Durchschnitt liegt bei 100 Quadratmetern.
2. Die Macher von Offenen Werkstätten verstehen sich verbreitet selbst als einzigartige Individuen, die sich nicht auf Funktionsrollen oder Klassifizierungsmerkmale reduzieren lassen wollen.

3. Offene Werkstätten sind singulär arrangierte Identifikationsräume für die in ihnen tätigen Personen.

4. Viele Offene Werkstätten zelebrieren Workshops oder Festivals als besondere, auch wiederkehrende, Ereignisse mit hohem Eigenwert.

\section{In der Gesellschaft der Singularitäten verschiebt sich das Produktionsparadigma von der Massenfertigung zur „Massen-Individualisierung “.}

Der engere Personenkreis, definiert als diejenigen Aktiven, die den alltäglichen Betrieb der Werkstatt maßgeblich ermöglichen und gestalten, besteht aus 1-60 Personen, während der weitere Personenkreis (nicht regelmäßige Nutzer) 2-2.000 Personen umfasst. Im Durchschnitt ist der weitere Personenkreis mit 50 Personen fünfmal so groß wie der engere Kreis mit durchschnittlich zehn Personen. Alle Befragten sind in den Jahren 1991 bis 2015 den Offenen Werkstätten beigetreten, $63 \%$ jedoch erst zwischen 2013 und 2015. Diese Zuwachsraten zeigen die Dynamik dieses Phänomens. Die Qualifikation der engeren Nutzer zeigt, dass $43 \%$ über Studienabschlüsse in naturwissenschaftlichen Disziplinen verfügen, gefolgt von Abschlüssen in den Geistes-, Gesellschafts- und Wirtschaftswissenschaften und im Bereich Medien und Kunst (22\%).

Die Befragung hat ergeben, dass die Hauptmotivation in der Wissensvermittlung ( $87 \%$ ), dem praktischen Arbeiten und dem Interesse besteht, einen Beitrag zur gesellschaftlichen Transformation zu leisten (je 78\%). Gemeinschaftliche Arbeitsprozesse „am Fall“ (Einzelstuickfertigung, Reparatur) sind für die Befragten eher prägend als ökonomische Verwertungsinteressen. Im Kontrast zur Bedeutung des Umweltschutzes als Motiv (knapp 60\% halten ihn für sehr wichtig) sind 56\% aller in Offenen Werkstätten verarbeiteten Materialien neu oder neuwertig. Freie Ressourcen, wie Schrotträder oder frei verfügbare Naturprodukte (9\%) und Spenden gebrauchter Materialien (26\%) spielen eine geringere Rolle in der Materialbeschaffung.

Die qualitativen Antworten aus der Online-Befragung und Einblicke aus der teilnehmenden Beobachtung belegen, dass Offene Werkstätten enge Bezüge zu allen fünf Spielarten der Singularisierung nach Reckwitz (2017, S. 58-63) aufweisen:

1. In Offenen Werkstätten werden, wie bereits angeführt, besondere Objekte hergestellt, die selten und oft auch einzigartig sind (z. B. Tauschschränke aus alten Telefonzellen). Die Prosumenten valorisieren in ihrer offenen und kollaborativen Fabrikation diese Objekte als besonders.
5. Die Macher von Offenen Werkstätten verstehen sich als besondere Kollektive, die praktisches Tun, Motive, erlebte Geschichten und erhoffte Wirkungen (z. B. Eigenversorgung bei Unterbrechungen globaler Supply Chains, Sinnstiftung anstelle anonymen Arbeitens und Konsums) vereinen.

Die Praktiken der Singularisierung in Offenen Werkstätten schließen das Beobachten (z. B. Entdeckung des besonderen Objekts) und Bewerten (z. B. Wertschätzung ausgefallener Objekte), das Hervorbringen (z. B. gezielte Singularisierungsarbeit an Objekten) und Aneignen (z. B. durch subjektives Erleben des kollaborativ gestalteten Objektes) ein.

\section{Implikationen des Singularitätscharak- ters von Offenen Werkstätten für die TA}

Die Auswirkungen der Praktiken in Offenen Werkstätten sind in COWERK mit Methoden ermittelt worden, die auch in anderen TA-Projekten zur Fassung hoher Diversität zum Einsatz kommen: Typologisierung, Fallbeispiele und Skalierung der Wirkungen. Im Folgenden reflektieren wir für diese drei Modi der TA, inwieweit sie sich für das Fassen des Phänomens der Singularitäten am Beispiel von Offenen Werkstätten eignen.

\section{Typologisierung}

Aufbauend auf den Befragungsergebnissen aus COWERK wurden die Offenen Werkstätten typologisiert, um über sie gebündelt kommunizieren zu können und Grundlagen für ihre Nachhaltigkeitsbewertung zu schaffen. Den Ausgangpunkt für die Typologisierung bildete die Frage nach der ,Art der Produktionen“ in der Offenen Werkstatt, wobei die Ausprägungen „Herstellung von Neuem“, „Hacking und Upcycling“ und „Reparatur“ differenziert wurden.

Diese drei Ausprägungen spiegeln die, für Offene Werkstätten charakteristischen, hohen Eigenkomplexitäten nicht mehr wider, denn durch die Typologisierung werden nur ausgewählte 
Gruppenmerkmale von Offenen Werkstätten erfasst. Die Kombination verschiedener Gewerke, Materialien und Techniken unter einem Dach sowie die verschiedenen Arten der Produktion in einer Offenen Werkstatt verschwinden somit, obwohl sich Singularitäten qualitativ und absolut deutlich voneinander unterscheiden. Mit dieser an der Abschätzung der Umweltwirkungen orientierten Typologisierung sind die für Singularitäten charakteristischen Eigenschaften der Originalität und Rarität der Artefakte eliminiert. Eigenkomplexitäten als ein potenziell TA-relevantes Merkmal verschwinden aber natürlich nur in der wissenschaftlichen Darstellung, in der Realität hingegen sind sie weiterhin strukturbildend wirksam (Reckwitz 2017, S. 54-55).

\section{Fallbeispiele}

In COWERK wurden Innovationsbiografien für das Lastenfahrrad und die Saftpresse nachgezeichnet, um die Beziehungen zur kommerziellen Wirtschaft auszuloten. Innovationen finden sich häufig zunächst dezentral in Nischen und verbreiten sich unter bestimmten Voraussetzungen massenhaft (Anderson 2006).

Das erste Long-John-Lastenfahrrad wurde 1923 von der dänischen Firma Smith \& Co. entwickelt, hatte daraufhin zahlreiche kommerzielle Anwendungen und geriet dann wieder in Vergessenheit. Im autofreien Kopenhagener Stadtteil Christiania erlebte es durch den Zuschnitt auf die Bedürfnisse der Nutzer eine Renaissance, wurde landesweit in großer Stückzahl produziert und ist mittlerweile ein Design-Klassiker. Derzeit werden in Offenen Werkstätten zahlreiche angepasste, einzigartige Lastenfahrräder kreiert, während Akteure wie die Deutsche Post standardisierte Lastenfahrräder massenhaft einsetzen. Der Berliner Senat fördert den Kauf von Lastenfahrrädern als ,,echte Alternative zu Auto und Lieferwagen“ (SENUVK 2018).
Antizipation der Verbreitung von singulären Gütern wie Saftpressen, die eigenständige Entwicklungen aus der Maker-Szene sind, außergewöhnlich große Unsicherheiten, vom Flop bis zum Knüller kann jede Antizipation Plausibilität und Realismus beanspruchen. Wie die Geschichte des Lastenfahrrads zeigt, erhöhen sich die Aussichten auf massenhafte Verbreitung, wenn der Innovations- und Produktionsprozess vielschichtig sowohl auf kommerzieller wie auch offen-kollaborativer Ebene stattfindet.

Die gelungene Überraschung und die Wertzuschreibung von Singularitäten entziehen sich jedoch der gezielten Planung und Steuerung. Während der Nutzen eines Standardgutes einfach zu ermitteln ist, unterliegt er bei Singularitäten komplizierten Valorisierungsdiskursen und -techniken. In der „The Winner Takes the Most"-Realität kulturalisierter Aufmerksamkeitsmärkte (Reckwitz 2017, S. 171) schlagen u. a. offene Designwettbewerbe (Local Motors 2018), Prototypenparties (Team Prototypenparty 2018) und Maker-Plattformen, u. a. das von IBM mitentwickelte MakerNet (MakerNet 2018) Brücken zwischen offenen Kollaborationen und kommerziellen Akteuren.

\section{Skalierung der Wirkungen}

Die Zukunft eines jungen Phänomens wie der Offenen Werkstätten, mit seiner Maker-Kultur und seiner Aufskalierung, ist mit Unwägbarkeiten verbunden. Beispielsweise formuliert das Wuhan Fab Lab: „Half of China's GDP will be generated by makers in the Future" (zitiert nach Saunders und Kingsley 2016, S. 32). In Deutschland divergieren die Einschätzungen zum zukünftigen Potenzial Offener Werkstätten für Wirtschaft und Gesellschaft. Je nach Stakeholdergruppe erscheinen rund 10.000 Offene Werkstätten im Jahr 2030, das entspricht in etwa der durchschnittlichen Anzahl an Gemeinden in Deutschland, als zu hoch

\section{Die Logik der Singularitäten als eine Logik der Spätmoderne erfordert eine Reflexion und Präzisierung des herkömmlichen TA-Rüstzeugs.}

Nach Reckwitz sind Singularitätsgüter ,im Prinzip ungewisse Güter und kulturelle Märkte Nobody-knows-Märkte“ (2017, S. 157, Hervorhebungen im Original). Die schwierige Zukunftseinschätzung von neuartigen Artefakten zeigt sich am Beispiel von aus alten Waschmaschinen hergestellten Saftpressen. Durch die in Offenen Werkstätten produzierten Saftpressen können nicht genutzte Äpfel in Wert gesetzt werden und damit einen Teil zur regionalen Versorgung beitragen. Die Anzahl solcher Saftpressen ist derzeit verschwindend gering. Zudem ist ihre zukünftige kommerzielle Nutzung angesichts der in vielen Regionen schrumpfenden Obstanbauflächen ungewiss: Zu DDR-Zeiten wurden alleine im Havelländischen Obstanbaugebiet auf 11.000 Hektar Äpfel angebaut, inzwischen in ganz Brandenburg nur noch auf knapp 900 Hektar (MLUL 2018, S. 3). So birgt die bzw. als viel zu niedrig angesetzt (Erdmann und Dönitz 2018). Einigkeit besteht hinsichtlich des großen Potenzials von Offenen Werkstätten für die Vermittlung von MINT-Kompetenzen (Erdmann und Dönitz 2018).

Wenn ein neues Phänomen wie Offene Werkstätten auftaucht, dann greift die Abbildung der monetären Wertschöpfung und seiner Wechselbeziehungen mit anderen Wirtschaftszweigen zu kurz, denn die Arbeitsweise in Offenen Werkstätten schafft eine neue Wertschöpfungskultur (Erdmann und Dönitz 2018). Sobald Singularitäten ihre sozialen Nischen verlassen und gesellschaftlich strukturbildend wirken, verschärft sich durch die Anfeuerung der Sichtbarkeits-, Valorisierungs- und Affizierungsmärkte auch die Polarisierung der Verteilung von Gütern, Arbeitsverhältnissen, Klassen und Lebensstilen, sozia- 
len Räumen und politischen Positionen (Reckwitz 2017, S. 107110). Skalierung ist mithin nicht nur eine Frage der Antizipation von Diffusionsverläufen und der Qualifizierung ungewisser Wirkungszusammenhänge, sondern auch der Einschätzung transformativer Wirkungen im Zuge der schleichenden Kulturalisierung von Ökonomie und Gesellschaft.

\section{Desiderata für eine neue TA-Agenda über singuläre Phänomene}

Die wesentlichen Begriffe, Konzepte und Instrumente der Technikfolgenabschätzung entstanden in der industriellen Moderne, weshalb sie auch an verallgemeinerbaren Techniken mit ihren typischen Mustern, Klassifizierungen und Regeln orientiert sind. Die Logik der Singularitäten als eine Logik der Spätmoderne unter anderen (Reckwitz 2017, S. 47) erfordert eine Reflexion und Präzisierung des herkömmlichen TA-Rüstzeugs.

Der Blick auf Besonderheiten wird, auch in der TA, verstellt, indem durch Bildung von Klassen, Subsumption, Abstraktion, Rangfolgen oder Skalen (Reckwitz 2017, S. 65-66) die Relevanz der kleinen Zahl und der Einzigartigkeit reduziert wird. Wie das Beispiel Offener Werkstätten zeigt, sieht sich die empirische und prospektive Untersuchung von Singularitäten allerlei Schwierigkeiten gegenüber. Es besteht also ein erhöhter Bedarf, die Singularitätskompetenz, d.h. das Beobachten und Wahrnehmen des Besonderen, in der TA zu fördern. Im Hinblick auf die Typologisierung wird empfohlen, den Verlust des Besonderen im Prozess zu reflektieren, die folgenden Aussagen anhand besonderer Einzelbeispiele zu überprüfen oder aber die Besonderheit als eine explizite Dimension der Typologisierung zu variieren.

Während die klassische TA auf Einzeltechniken fokussiert, „Technik ernst nehmen“ (Böschen und Dewald 2018), geht es bei Offenen Werkstätten und anderen vernetzten Produktionsmodi eher darum, Konfigurationen von Techniken, Materialien, Akteuren sowie kulturellen Praktiken und Bedeutungszuschreibungen in neuen Infrastrukturen ernst zu nehmen.

In der digitalen Ökonomie sind Bedingungen gegeben, die sowohl die raschere Verbreitung von wenigen Hits, als auch die ökonomisch ebenso gewichtige und noch wichtiger werdende kumulierte massenhafte Verbreitung von Nischenprodukten fördern (Anderson 2006). Die Menge singulärer Güter wird durch die digitalen Technologien wie personalisierte Endgeräteinstellungen, individualisierte Werbung, 3-D-Druck und Smart Production der Losgröße 1 potenziert, wobei es zu einer außergewöhnlichen Dynamisierung und Streuung der Aufmerksamkeit kommt, ohne allerdings die exakte Verteilung auf Artefakte vorhersagen zu können (Reckwitz 2017, S. 158). Gehören die Analyse der Aufmerksamkeitsströme und die Identifizierung von Champions auf den neuen Attraktivitätsmärkten zu den neuen Aufgaben für die TA? Gegebenenfalls beschränkt die Affektivität des Jetzt in der Spätmoderne aber auch grundsätzlich eine systematische Zukunftsbearbeitung wie in der Moderne üblich (Erdmann 2016; Reckwitz 2017, S. 431).
Klare Wirkungszusammenhänge lassen sich für Offene Werkstätten in Bezug auf Bildung, Sozialitäten und Wissen der beteiligten Akteure belegen. Angesichts der Bruchlinien der Nachverfolgbarkeit von Wirkungsketten (wie in den Innovationsbiografien skizziert) stellt sich die Frage, wie die weichen Effekte der Singularitätsgüterproduktion in TA-Diskursen ernster zu nehmen und zu den harten Effekten wie Markterfolg oder Umwelteffekten in Beziehung gesetzt werden können. Die TA bedarf angesichts der Singularisierung zudem neuer methodischer Impulse, um betrachtete Systeme aus dem kulturalisierten Supersystem auszuschneiden und ihre Folgen im Hinblick auf die Kulturalisierung abzuschätzen.

Diese Desiderata für eine neue TA-Agenda über singuläre Phänomene erfordern erhebliche konzeptionelle Fortentwicklungen. Gelingen die Wahrnehmung des Besonderen als Relevantem, die Abbildung der Kulturalisierung von Wirtschaft und Gesellschaft und der realitätsangemessene Zuschnitt des betrachteten Systems aus dem Supersystem nicht, so könnte sich ein immer größerer und wichtigerer Ausschnitt des soziotechnischen Geschehens (Anderson 2006) einer sinnvollen TA schleichend entziehen. Mit der Rückbindung der TA an die Diagnose der Singularisierung der Gesellschaft haben wir versucht zu zeigen, dass Zeitdiagnosen zu wertvollen Einsichten und methodischen Impulsen für die TA führen können. Gerade in Zeiten der Missionsorientierung von Innovationen sind auch die fortwährend stattfindenden, ambivalenten gesellschaftlichen Prozesse wie die Singularisierung als strukturbildende Kräfte für die Technik in den Blick zu nehmen.

\section{Literatur}

Alpsancar, Suzana (2018): Technikfolgenabschätzung als Zeitdiagnose. Einsichten aus Günter Ropohls Programm. In: TATuP - Zeitschrift für Technikfolgenabschätzung in Theorie und Praxis 27 (1), S. 14-20. DOI: 10.14512/tatup.27.1.14. Anderson, Chris (2006): The long tail. Why the future of business is selling less of more. New York: Hyperion.

Bogner, Eva; Löwen, Ulrich; Franke, Jörg (2018): Bedeutung der zukünftigen Produktion kundenindividueller Produkte in Losgröße 1. In: Tobias Redlich, Manuel Moritz und Jens Wulfsberg (Hg.): Interdisziplinäre Perspektiven zur Zukunft der Wertschöpfung. Wiesbaden: Springer Gabler, S. 63-75. Böhme, Gernot (2016): Ästhetischer Kapitalismus. Berlin: Suhrkamp. Böschen, Stefan; Dewald, Ulrich (2018): TA als Kontextualisierungsexpertise. Zwischen einfachem und reflexivem Modus. In: TATuP - Zeitschrift für Technikfolgenabschätzung in Theorie und Praxis 27 (1), S. 34-39. D0I: 10.14512/ tatup.27.1.34.

Erdmann, Lorenz (2016): Realweltliche Dynamiken in der Zukunftsforschung. Schwache Signale, koevolutionäre Szenarien und Living Labs. Präsentation auf der Tagung Zukunftsforschung und Transformation, Berlin, 24. 11. 2016. Online verfügbar unter https://www.izt.de/fileadmin/izt35/erdmann.pdf, zuletzt geprüft am 17.09.2018.

Erdmann, Lorenz; Dönitz, Ewa (2018): Zukünfte für Offene Werkstätten: Antizipation neuer Wertschöpfungsmuster in einem Visioning-Prozess. In: Tobias Redlich, Manuel Moritz und Jens Wulfsberg (Hg.): Interdisziplinäre Perspektiven zur Zukunft der Wertschöpfung. Wiesbaden: Springer Gabler, S. 51-62. 
Frank, Daniel (2018): Spätmoderne oder die Explosion des Besonderen.

In: TATuP - Zeitschrift für Technikfolgenabschätzung in Theorie und Praxis 27 (2), S. 72-73. DOI: 10.14512/tatup.27.2.72.

Kohtala, Cindy; Hyysalo, Sampsa (2015): Anticipated environmental sustainability of personal fabrication. In: Journal of Cleaner Production 99, S. 333-344.

Koren, Yoram; Shpitalni, Moshe; Gu, Peihua; Hu, Jack (2015): Product design for mass-individualization. In: Procedia CIRP 36, S. 64-71.

Kornwachs, Klaus (2018): Technikfolgenabschätzung als technikwissenschaftliche Disziplin? Methodenmix und Modellbildung. In: TATuP - Zeitschrift für Technikfolgenabschätzung in Theorie und Praxis 27 (1), S. 46-51. D0I: 10.14512/ tatup.27.1.46.

Lange, Bastian; Domann, Valentin; Häfele, Valerie (2016): Wertschöpfung in offenen Werkstätten. Eine empirische Erhebung kollaborativer Praktiken in Deutschland. Schriftenreihe des IÖW 213/16. Berlin: IÖW.

Lange, Bastian (2017): Offene Werkstätten und Postwachstumsökonomien. Kollaborative Orte als Wegbereiter transformativer Wirtschaftsentwicklungen? In: Zeitschrift für Wirtschaftsgeographie 61 (1), S. 38-55.

Local Motors (2018): Autonomous for all of us. Online verfügbar unter https://localmotors.com/, zuletzt geprüft am 18. 09.2018.

MakerNet (2018): MakerNet. Connecting makers to markets. Online verfügbar unter http://www.makernet.global/, zuletzt geprüft am 18.09.2018.

MLUL - Ministerium für Ländliche Entwicklung, Umwelt und Landwirtschaft (2018): Apfelland Brandenburg. Online verfügbar unter https://mlul.brandenburg.de/media_fast/4055/Apfelland-Brandenburg.pdf, zuletzt geprüft am 11.09.2018.

Pfaller, Robert (2018): Erwachsenensprache. Über ihr Verschwinden aus Politik und Kultur. Frankfurt am Main: Fischer.

Reckwitz, Andreas (2017): Die Gesellschaft der Singularitäten. Zum Strukturwandel der Moderne. Berlin: Suhrkamp.

Ritzer, George (2017): Die McDonaldisierung der Gesellschaft. Köln: Herbert von Halem.

Ropohl, Günter (2012): Allgemeine Systemtheorie. Einführung in transdisziplinäres Denken. Berlin: edition sigma.
Saunders, Tom; Kingsley, Jeremy (2016): Made in China. Maker spaces and the search for mass innovation. Nesta, British Council. Online verfügbar unter https://www.nesta.org.uk/report/made-in-china-makerspaces-and-thesearch-for-mass-innovation/, zuletzt geprüft am 11.09.2018.

Simons, Arno; Petschow, Ulrich; Peuckert, Jan (2016): Offene Werkstätten nachhaltig innovativ? Potenziale gemeinsamen Arbeitens und Produzierens in der gesellschaftlichen Transformation. Schriftenreihe des IÖW 212/16. Berlin: IÖW.

SENUVK - Senatsverwaltung für Umwelt, Verkehr und Klimaschutz (2018): Lastenräderförderprogramm. Online verfügbar unter https://www.berlin.de/ senuvk/verkehr/politik_planung/rad/lastenraeder/, zuletzt geprüft am 11.09.2018.

Team Prototypenparty (2018): Die Prototypenparty, das Matching Event für Produktentwickler, Designer, Start-Ups, Hardware-Hacker und Maker. Online verfügbar unter https://prototypenparty.com/, zuletzt geprüft am 18. 09.2018.

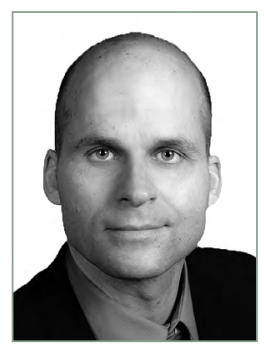

\section{LORENZ ERDMANN}

ist seit 2013 Leiter des Geschäftsfeldes Zukünfte und Gesellschaft am Competence Center Foresight des Fraunhofer-Instituts für System- und Innovationsforschung. Zu seinen Forschungsschwerpunkten gehören die Konzeption und Integration von Methoden der TA, der Nachhaltigkeits- und der Zukunftsforschung.

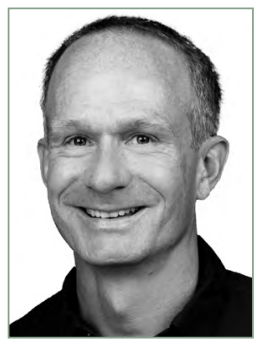

\section{PROF. DR. BASTIAN LANGE}

ist seit 2017 Privatdozent an der Universität Leipzig und war zwischen 2018-2019 Gastprofessor am Lehrstuhl für Wirtschaftsgeographie an der Universität Vechta. Zu seinen Forschungsschwerpunkten gehören alternative Ökonomien, Transitionsgeographien und neue Ansätze der Wirtschaftsgeographie und Governanceforschung.

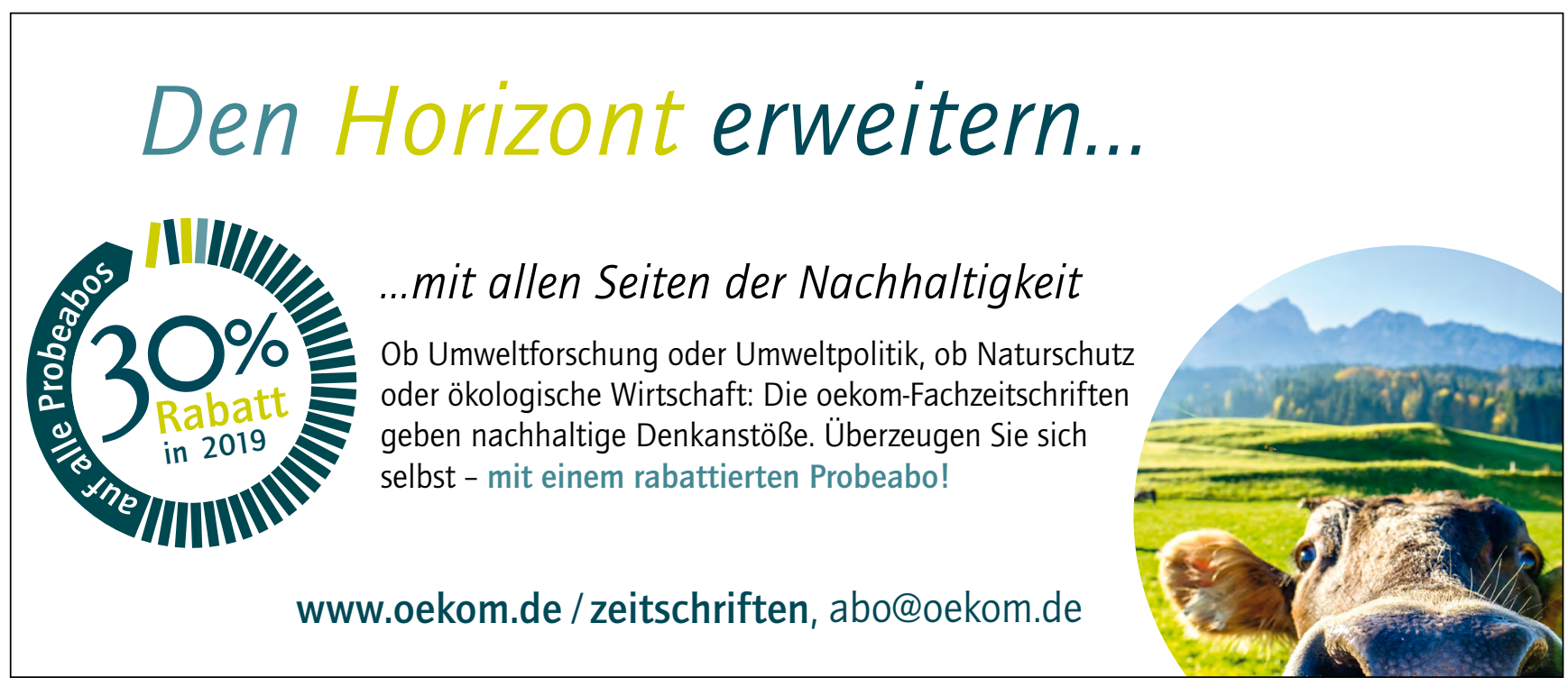

